\title{
Spin dynamic simulations of solid effect DNP: The role of the relaxation superoperator
}

\author{
Alexander Karabanov ${ }^{a}$, Grzegorz Kwiatkowski $^{a}$ and Walter Köckenberger ${ }^{a}$ \\ ${ }^{a}$ Sir Peter Mansfield Magnetic Resonance Centre, School of Physics and Astronomy, \\ University of Nottingham, UK; \\ (version 1)
}

Relaxation plays a crucial role in the spin dynamics of dynamic nuclear polarisation. We review here two different strategies that have recently been used to incorporate relaxation in models to predict the spin dynamics of solid effect dynamic nuclear polarisation. A detailed explanation is provided how the Lindblad-Kossakowski form of the master equation can be used to describe relaxation in a spin system. Fluctuations of the spin interactions with the environment as a cause of relaxation are discussed and it is demonstrated how the relaxation superoperator acting in Liouville space on the density operator can be derived in the LindbladKossakowski form by averaging out non-secular terms in an appropriate interaction frame. Furthermore we provide a formalism for the derivation of the relaxation superoperator starting with a choice of a basis set in Hilbert space. We show that the differences in the prediction of the nuclear polarisation dynamics that are found for certain parameter choices arise from the use of different interaction frames in the two different strategies. In addition we provide a summary of different relaxation mechanism that need to be considered to obtain more realistic spin dynamic simulations of solid effect dynamic nuclear polarisation.

Keywords: Lindblad-Kossakowski Equation; Liouville Space; Solid Effect Dynamic Nuclear Polarisation; Relaxation Superoperator; Spin Dynamics

\section{Introduction}

Dynamic nuclear polariation (DNP) can be used to substantially enhance the nuclear spin polarisation. There is currently substantial interest in the use of DNP by the magnetic resonance community since with the availability of robust hardware this strategy may help to overcome the sensitivity limitations of a wide range of applications including magic angle spinning NMR spectroscopy and magnetic resonance imaging. Several DNP pathways in solid state have been described in the literature. Depending on the number of electrons that interact with the same nuclei and the line width of the electron resonance spectrum solid effect DNP, cross effect DNP and DNP by thermal mixing have been distinguished. Solid effect dynamic nuclear polarisation (SE DNP) relies on i) a spin systems with negligible interactions between electrons so it is sufficient to consider only one electron interacting with an ensemble of nuclear spins. Nuclear spins are coupled to the electron through the hyperfine interaction and are also coupled through dipolar interaction between each other. ii) The electron linewidth is smaller than the Zeeman splitting of the nuclear spins. 


\section{The master equation}

Our objective is to calculate the dynamics of a model spin system consisting of one electron spin $S$ coupled by hyperfine interactions to nuclear spins $I_{k}$ during a constant irradiation with a microwave field. The general master equation for the density operator $\sigma$, in the frame rotating with the mw irradiation frequency $\omega_{0}$, is the Liouville von Neumann $(\mathrm{LvN})$ equation

$$
\frac{\mathrm{d}}{\mathrm{dt}} \sigma=-i \hat{H} \sigma-\hat{\Gamma} \sigma
$$

where $\sigma_{\text {th }}$ corresponds to the initial thermal equilibrium, $\hat{H}=[H, \cdot]$ is the Hamiltonian commutation superoperator and $\hat{\Gamma}$ is a relaxation superoperator whose form we need to specify later. We assume that the relaxation superoperator has been appropriately thermalised in such way that it ensures relaxation of the system back to the thermal equilibrium which is described by a density operator $\sigma_{t h}$. The Hamiltonian $H$ of the spin system consists of the following terms:

$$
H=H_{Z}+H_{I S}+H_{d}+H_{M W}=H_{0}+H_{M W},
$$

where the first term represents the Zeeman interaction of the electron and the nuclei with the external static magnetic field, the second term describes the hyperfine interaction between electron and nuclear spins and the third term represents the dipolar interaction between the nuclear spins. The fourth term represents the microwave irradiation applied orthogonally to the direction of the static magnetic field. The stationary Hamiltonian $H_{0}$ consists of only the interaction terms without the term arising from the microwave irradiation. The Zeeman interaction is defined by

$$
H_{Z}=\omega_{S} S_{z}+\omega_{I} \sum_{k} I_{k z}
$$

The hyperine interaction and the nuclear interaction can be written in terms of tensor products:

$$
H_{I S}=\sum_{k} I_{k} \cdot D_{k} \cdot S, \quad H_{I I}=\sum_{k \neq j} I_{k} \cdot d_{k j} \cdot I_{j}
$$

After transferring the master equation into a frame rotating with frequency $\omega_{0}$ we obtain

$$
H_{Z}=\Delta_{S} S_{z}+\omega_{I} \sum_{k} I_{k z}, \quad \Delta_{S}=\omega_{S}-\omega_{0}= \pm \omega_{I}
$$

The hyperfine interaction term of the Hamiltonian becomes

$$
H_{I S}=\sum_{k}\left(A_{0 k} S_{z} I_{k z}+\frac{1}{2} \sum_{k} A_{k+} S_{z} I_{k+}+\frac{1}{2} \sum_{k} A_{k-} S_{z} I_{k-}\right),
$$


and the dipolar interaction between nuclear spins can now be written in the truncated form:

$$
H_{d}=\sum_{j<k} d_{j k}\left(3 I_{j z} I_{k z}-I_{j} \cdot I_{k}\right) .
$$

Note that $H_{0}^{\prime}=H_{Z}+H_{I S}+H_{I I}$ is the truncated stationary Hamiltonian.

The irradiation of the spin system with microwaves close to the electron Larmor frequency is given by

$$
H_{M W}=\frac{\omega_{1}}{2}\left(S_{+}+S_{-}\right) .
$$

The appropriate Liouville space $\mathfrak{L}$ for this quantum mechanical problem is spanned by a basis using direct products of the single-spin unity operator, the Zeeman operators $\hat{I}_{k z}, \hat{S}_{z}$ and the rising and lowering operators $\hat{I}_{k \pm}, \hat{S}_{ \pm}$. The states can be classified either according to their coherence order or the correlation order of the basis operators For instance, the zero-quantum subspace $\mathfrak{L}^{0}$ contains all operators representating states with zero-quantum coherence irrespective of their spin correlation order while the three spin order subspace $\mathfrak{L}_{3}$ contains only operators that represent states in which three spins are correlated.

The number of spins that can be included in a coupled network in a quantum mechanical simulation of the solid effect are limited due to the exponential scaling of the dimensions of the Liouville space with the seize of the spin ensemble. A careful analysis of the participation of all states to the spin dynamics of the solid effect shows that mainly states belonging to the zero quantum coherence subspace contribute to it. All other states are only weakly populated. We have recently proposed to calculate an effective Hamiltonian based on an averaging procedure published by Krylov and Bogoliubov. The averaging procedure confines the dynamics of SE DNP to the zero quantum coherence subspace. A prerequisite for this strategy is the use of the Zeeman basis for the calculations.

A very important feature of DNP are relaxation processes that form the response of the spin system to the perturbation caused by the microwave irradiation. In combination, both the continuous irradation and the relaxation processes lead to an establishment of a quasi equilibrium for the spin system in which the population differences for the NMR transitions are enhanced in comparison to the thermal equilibrium state.

To maximise the number of spins in a model for SE DNP we want i) to reduce the required state space as much as possible while still obtaining a close approximation of the spin dynamics and ii) avoid the use of any operator diagonalisation since the required mathematical procedure impose a limitation of the quantum mechanical dimensions and thus the number of spins that can be included.

In the following we describe our approach to include relaxation in a model for SE DNP, keeping the above restrictions in mind. We discuss how our strategy fits into the general formalism for the description of dissipative quantum systems. Furthermore, we compare our approach to models proposed by others groups and discuss the differences in the model predictions that arise from the assumptions made in each of these models. 


\section{Lindblad-Kossakowsi relaxation superoperator}

If we use a specific form of the relaxation superoperator $\Gamma$ the Liouville von Neuman equation can be written in the so-called Lindblad-Kossakowski form

$$
\dot{\sigma}=-i \hat{H} \sigma-\hat{\Gamma} \sigma
$$

The commutation superoperator $\hat{H}$ represents again the Hamiltonian or coherent part of the dynamics and the superoperator $-\hat{\Gamma}$ describes the relaxation or decoherent part written as

$$
-\hat{\Gamma} \sigma=\sum_{k, j=1}^{N^{2}-1} C_{k j}\left[M_{k} \sigma M_{j}^{*}-\frac{1}{2}\left(\sigma M_{j}^{*} M_{k}+M_{j}^{*} M_{k} \sigma\right)\right]
$$

where $\left\{M_{k}\right\}_{k=1}^{N^{2}-1}$ is an orthonormal set of traceless operators in the $N$-dimensional Hilbert space and $\left(C_{k j}\right)$ is a positive matrix of relaxation rates. The operators $M_{k}$ stand for the coupling to the environment of the spin ensemble whose statistical properties are represented by the density operator $\sigma$. We refer to the term environment to describe interactions to the lattice and to other spins which have an effect on relaxation processes of the spin system under consideration. The LindbladKossakowski form guarantees that the master equation preserves the trace and the positiveness of the density operator for any initial value.

Due to the positiveness of $\left(C_{k j}\right)$, there exists always a unitary transformation

$$
L_{s}=\sum_{k=1}^{N^{2}-1} u_{s k} M_{k}, \quad s \in \overline{1, N^{2}-1}
$$

that leads to the diagonal form of the relaxation superoperator $\Gamma$, which in this form is frequently called the Lindbladian

$$
-\hat{\Gamma} \sigma=\sum_{k=1}^{N^{2}-1} \gamma_{k}\left[L_{k} \sigma L_{k}^{*}-\frac{1}{2}\left(\sigma L_{k}^{*} L_{k}+L_{k}^{*} L_{k} \sigma\right)\right] .
$$

Here $\left\{L_{k}\right\}_{k=1}^{N^{2}-1}$ is again an orthonormal set of traceless operators and $\gamma_{k}$ are nonnegative rates. Note that the condition of normalization for $L_{k}$ is actually not necessary, because any normalization can be achieved by a suitable choice of the rates $\gamma_{k} \geq 0$.

The set $\left\{L_{k}\right\}$ and the rates $\gamma_{k}$ (or the set $\left\{M_{k}\right\}$ and the rates $C_{k j}$ ) can be specified if a choice is made in respect to the origin of the relaxation mechanisms that cause transitions between populations and the loss of coherences in the quantum system.

\section{The spin interaction frame}

As already shown by Redfield relaxation of a spin ensemble under a periodic perturbation can differ from the situation when the system relaxes back to the thermal state $\sigma_{t h}$ after the perturbation has stopped. Formally this can be taken into account by transferring the master equation into the rotating frame precessing at the frequency of the time dependent perturbation. For the simulation of the evolution of the spin ensemble under continuous microwave irradiation, we have to proceed 
to the frame rotating with the microwave frequency $\omega_{0}$. In this frame, the effective part of the Liouvillian is the part commuting with the Zeeman component $S_{z}$ of the electronic spin. Furthermore, at the solid effect resonance $\omega \pm \omega_{I}$, effectively the polarization dynamics is reduced to the subspace of operators commuting with the resonant part of the Zeeman interaction.

Thus, the dynamics is described in the spin interaction frame,

$$
\sigma=e^{-i \hat{H}_{Z} t} \bar{\sigma}, \quad \hat{H}_{Z} \equiv\left[H_{Z}, \cdot\right], \quad H_{Z}=\omega_{S} S_{z}+\omega_{I} \sum I_{k z}
$$

where the higher order Krylov-Bogolyubov method should be used to accurately average out non-secular terms. The effective relaxation superoperator in this case commutes with the commutation superoperator $\hat{H}_{Z}$. This imposes certain restrictions on the set $\left\{L_{k}\right\}$ of the Lindblad-Kossakowski operators. The microwave driven dynamics is no longer the free evolution at static field, so the Lindbladian (3) no longer describes the free thermal relaxation and should be chosen in a way consistent with the dynamics in the interaction frame.

It is possible to show (see Appendix 11.1) that the relaxation superoperator in the Lindblad-Kossakowski form is invariant to a transformation into the interaction frame if the set of Lindblad-Kossakowski operators $L_{k}$ are an orthogonal set of traceless eigenoperators of the Zeeman superoperator $\hat{H}_{Z}$.

\section{Fluctuations as the origin of relaxation}

The origin of relaxation in a spin system are fluctuations of the interactions of the spins with their environment. These fluctuations can modulate interactions with surrounding spins and the lattice. We can add to the stationary Hamiltonian $H_{0}$ of a quantum system a random time-dependent term $H_{f}(t)$ to account for the fluctuations.

$$
H=H_{0}+H_{f}(t)
$$

The fluctuating part is represented by the Hermitian operator $H_{f}(t)$ whose matrix elements describe random stationary processes with zero averages. The relaxation superoperator can be found in the following three steps (ref: Bloembergen, Purcell and Pound, see also Abragam, Redfield, Goldman and others).

First, we proceed to the Zeeman (spin interaction) frame by the rule

$$
H_{f}(t) \quad \longrightarrow \quad H_{f}^{\prime}(t)=e^{i H_{Z} t} H_{f}(t) e^{-i H_{Z} t}
$$

where $H_{Z}$ denotes the Zeeman part of the unperturbed Hamiltonian $H_{0}$. Second, we calculate the second order approximation leading to the following doublecommutator superoperator

$$
\left.\hat{\Gamma}(t)=\frac{1}{2} \int_{-\infty}^{+\infty} \overline{\left[H_{f}^{\prime}(t),\left[H_{f}^{\prime}(t-\tau)\right.\right.}, \cdot\right] d \tau
$$

where the overline means the temporal ensemble average. Third, non-secular terms in $\hat{\Gamma}(t)$ should be neglected by taking the time average

$$
\hat{\Gamma}(t) \quad \longrightarrow \quad \hat{\Gamma}_{0}=\langle\hat{\Gamma}(t)\rangle \equiv \lim _{t \rightarrow+\infty} \frac{1}{t} \int_{0}^{t} \hat{\Gamma}(t) d t .
$$


It is important to note that when calculating the time average (7), it is assumed that the minimal energy difference between eigenvalues of $H_{Z}$ is much larger than the maximal relaxation rate caused by the fluctuating part. This is usually the case at high magnetic field when working in the Zeeman basis. For example, in the SE DNP case the eigenvalues of $H_{Z}$ are

$$
\Omega_{p q}=p \omega_{I}+q \omega_{S}, \quad|p| \leq n, \quad|q| \leq 1,
$$

where the sum $p+q$ provides the coherence order of the corresponding eigenstates. The minimal energy difference between the eigenvalues (at least in the subspaces with less than 500-quantum coherences) is $\left|\omega_{I}\right|$. At typical high field, this is much larger than any relaxation rate observed in experiments. In this respect, the Zeeman frame (5) is universal and provides the maximal elimination of non-secular terms. It is instructive to analyse whether it is possible to use a different frame instead of the Zeeman frame to average out the non-secular terms in $\hat{\Gamma}(t)$. In the frame corresponding to the rotation defined by an arbitrary Hermitian operator $H$, we make the change

$$
\sigma \rightarrow \sigma^{\prime}=e^{i H t} \sigma e^{-i H t}
$$

Let $\sigma_{k j}^{\prime}$ be the matrix elements of $\sigma^{\prime}$ in the basis of eigenstates $\lambda_{k}$ of $H$,

$$
\sigma_{k j}^{\prime}=\left\langle\lambda_{k}\left|\sigma^{\prime}\right| \lambda_{j}\right\rangle
$$

According to the fluctuations approach, under the action of the relaxation superoperator, the matrix element $\sigma_{k j}^{\prime}$ changes in time as (ref: Abragam's Principles, Chapter VIII, section $\mathrm{C}$ where the random fluctuations approach is decribed):

$$
\frac{d}{d t} \sigma_{k j}^{\prime}=\sum_{k^{\prime}, j^{\prime}} e^{i \Omega_{k j, k^{\prime} j^{\prime}} t} R_{k j, k^{\prime} j^{\prime}} \sigma_{k^{\prime} j^{\prime}}^{\prime}, \quad \Omega_{k j, k^{\prime} j^{\prime}}=\lambda_{k}-\lambda_{j}-\lambda_{k^{\prime}}+\lambda_{j^{\prime}}
$$

where $R_{k j, k^{\prime} j^{\prime}}$ are some time-independent relaxation rates. The terms with

$$
\Omega_{k j, k^{\prime} j^{\prime}} \neq 0
$$

are called non-secular terms. For $k^{\prime}, j^{\prime}$ such that

$$
\left|\frac{R_{k j, k^{\prime} j^{\prime}}}{\Omega_{k j, k^{\prime} j^{\prime}}}\right| \ll 1
$$

the time-dependence in (8) is fast oscillating, and the corresponding non-secular terms in the sum can be neglected. Otherwise, terms with $k^{\prime}, j^{\prime}$ such that the rate $R_{k j, k^{\prime} j^{\prime}}$ is of the same order of magnitude or larger than the eigenvalue $\Omega_{k j, k^{\prime} j^{\prime}}$ are not fast oscillating. Their effect on the spin dynamics of the spin system can be appreciable, so they cannot be neglected.

All non-secular terms can be removed in the case when

$$
\forall k, j, k^{\prime} j^{\prime} \quad \Omega_{k j, k^{\prime} j^{\prime}}=0 \quad \text { or } \quad\left|\frac{R_{k j, k^{\prime} j^{\prime}}}{\Omega_{k j, k^{\prime} j^{\prime}}}\right| \ll 1 .
$$


Condition (9) is violated if there exist eigenstates of $H$ with differences between the eigenvalues much smaller than the corresponding relaxation rates. For example, if

$$
0 \neq\left|\lambda_{k}-\lambda_{j}\right| \ll\left|R_{k j, j k}\right|
$$

then

$$
\Omega_{k j, j k}=2\left(\lambda_{k}-\lambda_{j}\right) \neq 0, \quad\left|\frac{R_{k j, j k}}{\Omega_{k j, j k}}\right| \gg 1 .
$$

Thus, not any operator $H$ can be used in the removal of the non-secular terms and not any rates $R_{k j, k^{\prime} j^{\prime}}$ can be chosen except those for which condition (9) is satisfied. This condition is always satisfied if $H=H_{Z}$, because the smallest nonzero difference between the eigenvalues of $H_{Z}$ is $\left|\omega_{I}\right|$, which is always much bigger than any relaxation rates in the system.

We show in the Appendix 11.2 that based on the assumption that $H_{f}$ is built of a full set $\left\{F_{p q, m}\right\}$ of orthonormal eigenvectors of $\hat{H}_{Z}$ it is always possible to derive the relaxation superoperator $\Gamma^{\prime}$ in the Lindblad-Kossakowski form.

\section{Uncorrelated random field model}

As the simplest model for a relaxation mechanism we can consider uncorrelated fluctuations of the local magnetic field (along the three spatial directions with no specific preference). The assumption of this model determines the choice of the set $\left\{L_{k}\right\}$ of Lindblad-Kossakowski operators for which the set of single-spin order operators written in terms of Zeeman components and lowering and raising operators can be used,

$$
\left\{L_{k}\right\}=\left\{S_{z}, S_{ \pm}, I_{s z}, I_{s \pm}, s \in \overline{1, n}\right\} .
$$

Starting from this set of traceless operators the relaxation superoperator can be built (see Appendix 11.3) and we get

$$
\begin{gathered}
\hat{\Gamma} \sigma=R_{2} \hat{S}_{z}^{2} \sigma+R_{1}\left(\hat{S}_{+} \hat{S}_{-}+\hat{S}_{-} \hat{S}_{+}\right) \sigma+R_{3}\left(S_{+} \sigma S_{-}-S_{-} \sigma S_{+}+\sigma S_{z}+S_{z} \sigma\right)+ \\
+\sum_{k=1}^{n}\left[r_{2 k} \hat{I}_{k z}^{2} \sigma+r_{1 k}\left(\hat{I}_{k+} \hat{I}_{k-}+\hat{I}_{k-} \hat{I}_{k+}\right) \sigma+r_{3 k}\left(I_{k+} \sigma I_{k-}-I_{k-} \sigma I_{k+}+\sigma I_{k z}+I_{k z} \sigma\right)\right]
\end{gathered}
$$

where $R_{j}, r_{j k}$ are some rates to be specified.

At cryogenic temperatures of about $1 \mathrm{~K}$ typical for DNP and modest magnetic fields of $3.5 \mathrm{~T}$, the thermal density operator is well approximated using only the Zeeman part of the stationary Hamiltonian,

$$
\sigma_{t h}=\frac{e^{-\beta H_{0}}}{\operatorname{Tr} e^{-\beta H_{0}}} \sim \sigma_{t h}^{\prime}=\frac{e^{-\beta H_{Z}}}{\operatorname{Tr} e^{-\beta H_{Z}}}, \quad \beta=\frac{\hbar}{k_{b} T}
$$

Furthermore, since $\left|\omega_{S}\right| \gg\left|\omega_{I}\right|$, the following approximation can be used

$$
\sigma_{t h}^{\prime} \sim \frac{1}{N}\left(1-2 p_{0} S_{z}\right), \quad p_{0}=\tanh \frac{\beta \omega_{S}}{2} .
$$


We can assume that

$$
\hat{\Gamma} S_{z}=\frac{1}{T_{1 e}} S_{z}, \quad \hat{\Gamma} S_{ \pm}=\frac{1}{T_{2 e}} S_{ \pm}
$$

where $T_{1 e}, T_{2 e}$ are the longitudinal and transverse relaxation times of the electron, that can be experimentally obtained. Relaxation does not affect the thermal equilibrium, $\hat{\Gamma} \sigma_{t h}^{\prime}=0$, hence we obtain

$$
R_{1}=\frac{1}{4 T_{1 e}}, \quad R_{2}=\frac{1}{T_{2 e}}-\frac{1}{2 T_{1 e}}, \quad R_{3}=\frac{p_{0}}{2 T_{1 e}}, \quad r_{3 k}=0 .
$$

Note that the two terms in (10) with the two factors $R_{3}$ or $r_{3 k}$ ensure that the spin system relaxes back to the thermal state $\sigma_{t h}$. Using the experimental nuclear longitudinal and transverse relaxation times $T_{1 n, k}, T_{2 n, k}$, the remaining rates can be obtained as

$$
r_{1 k}=\frac{1}{4 T_{1 n, k}}, \quad r_{2 k}=\frac{1}{T_{2 n, k}}-\frac{1}{2 T_{1 n, k}} .
$$

The relaxation superoperator (10) with the rates (11), (12) corresponds to the uncorrelated random field relaxation model adapted to the thermal relaxation in the spin interaction frame. At this point it is important to note that we could have chosen a more complex relaxation model which includes fluctuations of the hyperfine interaction between electrons and nuclear spins. In this case we would have to include also second order spin correlation operators to built the corresponding relaxation superoperator. We will discuss such more complex models in a later section.

\section{Constructing the relaxation superoperator starting from a basis set in Hilbert space}

In this section we discuss a different way to derive the relaxation superoperator in the Lindblad-Kossakowski form. We select a set of basis vectors in Hilbert space, construct a set of elementary traceless operators and build the relaxation superoperator in the corresponding Liouville space. Furthermore, we introduce relaxation rates for both longitudinal relaxation and transverse relaxation without using any specific relaxation model. The motivaton for this analysis is a recent string of publications by the Vega group using a related concept to include relaxation in DNP simulations.

First we choose an orthonormal basis $\left\{v_{s}\right\}_{s=1}^{N}$ of the Hilbert space. As a specific example this could be the eigenbasis of the stationary Hamiltonian $H_{0}$. As the Zeeman part $H_{Z}$ of the Hamiltonian is diagonal in the eigenbasis of the stationary Hamiltonian $H_{0}$ the choice of this basis fulfils the requirement of section 4 . Consider the set of operators in the Hilbert space

$$
\left\{O_{s s^{\prime}} \equiv v_{s} v_{s^{\prime}}^{*}\right\}_{s, s^{\prime}=1}^{N}
$$

The $\left(s s^{\prime}\right)$-matrix element of the operator $O_{s s^{\prime}}$ in the basis $\left\{v_{s}\right\}$ equals 1 and all other elements are zero, so the set $\left\{O_{s s^{\prime}}\right\}$ is orthogonal in the trace norm. The non-diagonal subset $\left\{O_{s s^{\prime}}, s \neq s^{\prime}\right\}$ is traceless and hence fulfils the condition for Lindblad operators. For the diagonal operators contained in the subset $\left\{O_{s s}\right\}$ the 
trace is always 1 . However, we can use this subset as an orthogonal basis to construct from linear combinations of the operators $O_{s s}$ a new set of traceless operators

$$
O_{q}=\sum_{s=1}^{N} c_{q s} O_{s s}, \quad q \in \overline{1, N-1},
$$

where due to requirement of the tracelessness and othogonality the coefficients $c_{q s}$ must satisfy the conditions

$$
\forall q \quad \sum_{s=1}^{N} c_{q s}=0, \quad \forall q \neq q^{\prime} \quad \sum_{s=1}^{N} c_{q s} c_{q^{\prime} s}^{*}=0 .
$$

Using this strategy we can construct a complete orthogonal set of traceless operators

$$
\left\{L_{k}\right\}=\left\{O_{s s^{\prime}}\right\}_{s \neq s^{\prime}=1}^{N}+\left\{O_{q}\right\}_{q=1}^{N-1}
$$

that we can use to build the relaxation superoperator in the Lindblad-Kossakowski form: ${ }^{1}$

$$
\begin{gathered}
-\hat{\Gamma}_{0} \sigma=\sum_{s \neq s^{\prime}} \Gamma_{s s^{\prime}}\left[O_{s s^{\prime}} \sigma O_{s^{\prime} s}-\frac{1}{2}\left(\sigma O_{s^{\prime} s^{\prime}}+O_{s^{\prime} s^{\prime}} \sigma\right)\right]+ \\
+\sum_{q} \Gamma_{q}\left[O_{q} \sigma O_{q}^{*}-\frac{1}{2}\left(\sigma O_{q}^{*} O_{q}+O_{q}^{*} O_{q} \sigma\right)\right] .
\end{gathered}
$$

Note that this expression corresponds to the definition of the diagonal Lindbladian in (3). We can conclude, that in principle, it is possible to derive the relaxation superoperator in the Lindblad form by choosing a basis set but without selecting first a relaxation model as we did in section 6 . As a consequence, no formal time averaging of non-secular terms is required in the derivation of the time independent Lindbladian (14). However, as we will discuss in more details in the next section further below, an implicit assumption was made in the derivation of the Lindbladian that the frame fixed by the choice of the basis enables the full removal of non-secular terms and condition (9) in Section 5 is fulfilled.

The full set of the elementary operators $O_{s s^{\prime}}$ generated by the basis $\left\{v_{s}\right\}$ can be used as an orthonormal basis in the Liouville operator space. It is now possible to introduce rates that describe the changes of the states represented by the operators $O_{s s^{\prime}}$ due to relaxation. The action of the relaxation superoperator on a state $O_{s s}$ corresponds to a change of populations in Hilbert space. Therefore we associate a rate $R_{1}$ for longitudinal relaxation with it. Correspondingly, the action of the relaxation superoperator on a state specified by $O_{s s^{\prime}}, s \neq s^{\prime}$ describes the loss of a coherence in Hilbert space and therefore we associate a rate $R_{2}$ for transverse relaxation with this process. It follows from the form (14) that

$$
\hat{\Gamma}_{0} O_{k k}=\sum_{s \neq k} R_{1, s k}\left(O_{k k}-O_{s s}\right), \quad \hat{\Gamma}_{0} O_{k j}=R_{2, k j} O_{k j}, \quad k \neq j
$$

\footnotetext{
${ }^{1}$ see Appendix 11.5 for a detailed explanation how to implement this formalism numerically
} 


$$
R_{1, s k}=\Gamma_{s k}, \quad R_{2, k j}=\frac{1}{2}\left[\sum_{s \neq k} \Gamma_{s k}+\sum_{s \neq j} \Gamma_{s j}+\sum_{q} \Gamma_{q}\left(\left|c_{q k}\right|^{2}+\left|c_{q j}\right|^{2}-2 c_{q k} c_{q j}^{*}\right)\right] .
$$

To ensure that the spin system relaxes back to the thermal state $\sigma_{t h}$ we have to weight the rates with the appropriate Boltzmann factors. The density operator at thermal equilibrium in the Boltzmann statistics is given by the formula

$$
\sigma_{t h}=\frac{e^{-\beta H_{0}}}{\operatorname{Tr} e^{-\beta H_{0}}}, \quad \beta=\frac{\hbar}{k_{b} T} .
$$

The relaxation superoperator acts trivially on $\sigma_{t h}$. Using the expansion

$$
\sigma_{t h}=\sum_{k, j=1}^{N} p_{k j} O_{k j}
$$

we obtain then

$$
0=\hat{\Gamma}_{0} \sigma_{t h}=\sum_{k \neq j} p_{k j} R_{2, k j} O_{k j}+\sum_{k} p_{k k} \sum_{s \neq k} R_{1, s k}\left(O_{k k}-O_{s s}\right) .
$$

This gives

$$
\forall k \neq j \quad p_{k j} R_{2, k j}=0, \quad p_{k k} R_{1, j k}-p_{j j} R_{1, k j}=0 .
$$

As long as conditions (13), (17) are satisfied and no additional conditions are imposed on the spin dynamics, the choice of the basis $\left\{v_{s}\right\}$ and the coefficients $c_{q s}$, defining the set $\left\{L_{k}\right\}$ of Lindblad-Kossakowski operators, and the non-negative rates $\Gamma_{s s^{\prime}}, \Gamma_{q}$ can be arbitrary. For example, it is feasible to use the eigenvectors of the time-independent Hamiltonian $H_{0}$ to construct the Lindblad-Kossakowski operators, built the relaxation superoperator and choose a set of rates. Formulas (15) in conjunction with the definitions of the rates in Vega's paper can be used to reproduce the relaxation superoperator as defined in their paper.

It is noteworthy that this strategy works for only specific choices of the elementary operator set $O_{s s^{\prime}}$. For instance, it is not possible to define a basis $\left\{v_{s}\right\}$ in Hilbert space that can be used to construct the operators

$$
S_{z}, S_{ \pm}, I_{s z}, I_{s \pm}, s \in \overline{1, n}
$$

which we have used as a set of Lindblad-Kossakowski operators to derive the relaxation superoperator based on the uncorrelated random field model. This is due to the fact that the raising and lowering operators can only be defined as combinations of elementary operators $O_{s s^{\prime}}$ in the Zeeman basis.

\section{Comparison between the different strategies}

We compare now in more details the two different strategies to incorporate relaxation in a spin dynamics model for SE DNP, which we have introduced in the last two sections. The motivation for this comparison arises from the different mathematical operations that these strategies require. The uncorrelated random field strategy relies on the choice of a relaxation model (such as uncorrelated 
random fluctuations) and the assumption that mixing of the states due to the pseudosecular part of the hyperfine interaction is negligible for the derivation of the relaxation superoperator. The positive feature is that the Zeeman basis can be used for the spin dynamics calculations and that a diagonalisation of the stationary Hamiltonian $H_{0}$ is not required. This provides a particular advantage when trying to simulate the dynamics of quantum systems containing many coupled spins since diagonalisation becomes impossible for the large matrices that appear in these simulations. On the other hand working in the eigenbasis of the stationary Hamiltonian has the advantage that any mixing of states due to the non-secular part of the hyperfine interaction is accounted for and the diagonal density operator in Hilbert space provides directly the populations of the different energy levels. There is apparently no need to formally average out non secular terms arising from the assumption of a fluctuation model.

In general, the uncorrelated random field model in the interaction frame (10) built of eigenoperators of the commutation superoperator $\hat{H}_{Z}$ and the model-free strategy (14) based on eigenoperators of the commutation superoperator $\hat{H}_{0}$ are inconsistent, because both relaxation superoperators are diagonal in the form (3) but use different Lindblad-Kossakowski operator sets $\left\{L_{k}\right\}$. The former uses single-spin orders $S_{z}, S_{ \pm}, I_{k z}, I_{k \pm}$ according to the uncorrelated random field model, the later uses the elementary operators $O_{k j}$ in the basis of eigenstates of the stationary Hamiltonian $H_{0}$. Expectedly, the spin dynamics described by these two, in principle different models, will be different.

Apart from the choice of the bases there is also a subtle but crucial difference in respect of the use of the interaction frame. If the eigenstates of the stationary Hamiltonian $H_{0}$ are used as a basis and no transformation of the Lindbladian into the interaction frame is carried out, an assumption is made that the relaxation processes during microwave irradiation is equivalent to free relaxation of the spin system after it was perturbed by a driving field. Using a truncated Hamiltonian $H_{0}^{\prime}$ as it is done by Vega will eliminate this issue. A more important issue becomes evident when using the truncated stationary Hamiltonian $H_{0}^{\prime}$ instead of the Zeeman Hamiltonian $H_{Z}$ as a reference frame to derive the relaxation superoperator using the random fluctuations model. For this reference frame it can be shown that for certain choices of parameters, that lead to strong mixing of the Zeeman states and high transverse relaxation rates, the condition (9) is not always fulfilled. This has the consequence that not all non-secular terms can be averaged out. This issue is not immediately apparent when following the strategy described in section 7 since non-secular terms do not appear in the formalism. However, it makes this formalism inconsistent with a relaxation model based on fluctuating interactions between spins and their environment.

We present now a set of numerical simulations to illustrate our analysis. For the simplest two spin system consisting of one electron and one nuclear spin (1e 1n) there is always a good agreement between the model (14) when using the eigenbasis of the truncated Hamiltonian $H_{0}^{\prime}$ described in section 7 and the model based on the uncorrelated field fluctuations (10) provided that we assume random fluctuations along all three spatial directions. ${ }^{1}$ Note that in the Vega paper only fluctuations along the x-directions are assumed.

\footnotetext{
${ }^{1}$ Basically we have to calculate $\frac{1}{T_{1, j k}}=\frac{1}{T_{1 e}}\left|\left\langle\lambda_{k}\left|S_{x}\right| \lambda_{j}\right\rangle\right|^{2}+4 \sum_{i} \frac{1}{T_{1 n, i}}\left|\left\langle\lambda_{k}\left|I_{x}\right| \lambda_{j}\right\rangle\right|^{2}+\frac{1}{T_{2 e}}\left|\left\langle\lambda_{k}\left|S_{z}\right| \lambda_{j}\right\rangle\right|^{2}+$ $4 \sum_{i} \frac{1}{T_{2 n, i}}\left|\left\langle\lambda_{k}\left|I_{z}\right| \lambda_{j}\right\rangle\right|^{2}$
} 

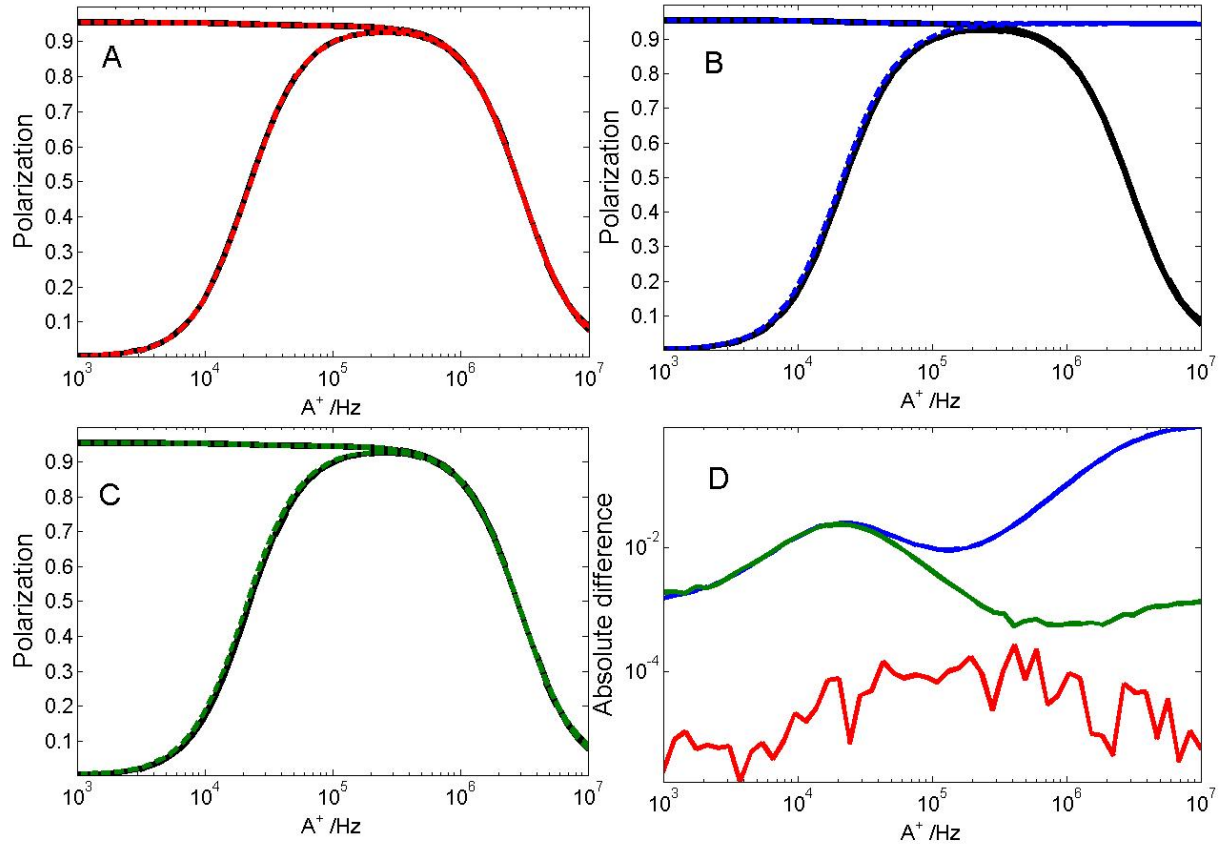

Figure 1. Simulation of the nuclear steady state polarisation for a system of one electron with one nuclear spin. The strength of the pseudosecular interaction $A_{+}$is changed between $1 \mathrm{kHz}$ and $10 \mathrm{MHz}$. A: Comparison between uncorrelated random fluctuation model (10) (black) and the strategy (14) that involves the choice of the eigenbasis $H_{0}^{\prime}$ (red).B: Comparison between random fluctuation model (10) (black) and original Vega's model with fluctuations only assumed along $I_{x}$ (blue). C: Comparison between random fluctuation model (10) (black) and Vega's model with fluctuations also assumed along $I_{z}$ (green). D: difference between the simulations. Red refers to A, blue to B and green to C. Note the logaritmic scale and the large error between simulations in $\mathrm{C}$ that disappears when fluctuations along $I_{x}$ and $I_{z}$ are assumed in Vega's model. Model parameters: $\omega_{I}=144 \mathrm{MHz}, \mathrm{MW}=0.1 e 6, R_{1 e}=1, R_{2 e}=1 e 5, r_{1 n}=1 e-2, r_{2 n}=1 e 4$

Figure (1) demonstrates that for the simple 2 spin systems (1e 1n) there is good agreement between the random fluctuation model $\Gamma(10)$, the model-free strategy $\Gamma_{0}$ explained in section 7 (14) and Vega's original simulations provided that we modify his rates in such a way that also fluctuations along $I_{z}$ are allowed. This modification of his model is essential to get good agreement with the random fluctuation model also for more than one nuclear spin.

For the case that the model spin system consists of several nuclear spins and one electron spin we demonstrate now that there could be a substantial discrepancy in the spin dynamics predictions depending on the strength of the nuclear dipolar interaction in comparison to the difference of the strength of the secular term of the hyperfine interactions of the nuclei with the electron.

We use as an example a model spin system consisting of one electron and two nuclear spins. Suppose that, calculating the basis of eigenstates of $H_{0}$, we can neglect terms, not commuting with $H_{Z}$, that is we assume $\left|B_{k}\right| \ll\left|\omega_{I}\right|$. This leads to the simplified stationary Hamiltonian

$$
H_{0}^{\prime}=H_{Z}+\sum_{k<j} d_{k j}\left(2 I_{k z} I_{j z}-\frac{1}{2} I_{k+} I_{j-}-\frac{1}{2} I_{k-} I_{j+}\right)+\sum_{k} A_{k} I_{k z} S_{z}
$$




\subsection{The case when the nuclear dipolar interaction is quenched by the hyperfine interaction - core nuclei}

First we consider the case when the dipolar interaction between the nuclei is much smaller than the difference between the strength of the secular terms of the hyperfine interaction that describes their coupling to the elctron spin. The nuclei are in this case close to the electron and the dipolar interaction between them is quenched by their coupling to the electron. In DNP models such nuclear spins belong to the core.

$$
\forall k \neq j \quad \frac{\left|d_{k j}\right|}{\left|A_{k}-A_{j}\right|} \ll 1
$$

Under these conditions the nuclear interaction term is negligible and the basis of eigenstates of $H_{0}^{\prime}$ is well approximated by the Zeeman basis $\left\{v_{s}\right\}$. We show in the Appendix 11.4 that in this case the two models can be modified in such a way that their predictions of the SE DNP spin dynamics are very close. Using the additional conditions that the transverse relaxation time constants are much shorter than the longitudinal time constants

$$
\frac{1}{T_{2 e}}, \frac{1}{T_{2 n, k}} \ll \frac{1}{T_{1 e}}, \frac{1}{T_{1 n, k}}, \quad \forall k \neq j \quad \frac{\left|d_{k j}\right|}{\left|A_{k}-A_{j}\right|} \ll 1
$$

the model based on the uncorrelated random fluctuations $\hat{\Gamma}$ and the model $\hat{\Gamma}_{0}$ based on the choice of the eigenbasis of $H_{0}^{\prime}$ can be made close to each other if we let

$$
\begin{gathered}
\hat{\Gamma}_{0} O_{k j}=R_{2, k j} O_{k j}, \quad R_{2, k j}=\operatorname{Tr}\left(\left(\hat{\Gamma} O_{k j}\right) O_{j k}\right), \quad k \neq j, \\
\hat{\Gamma}_{0} O_{k k}=\sum_{j \neq k} R_{1, k j}\left(O_{k k}-O_{j j}\right), \quad R_{1, k j}=-\operatorname{Tr}\left(\left(\hat{\Gamma} O_{k k}\right) O_{j j}\right) .
\end{gathered}
$$

We show the good agreement for the two models (10) and (14) in Figure (2)A and C. Note that the absolute error between the two simulations is very small.

\subsection{The case when the dipolar interaction is not quenched by the hyperfine interaction - the bulk nuclei}

If the nuclei are relatively far away from the electron the nuclear dipolar interaction can be larger than the difference between the strengths of the secular hyperfine interactions of the nuclei. Such conditions can be found for bulk nuclei. The first of conditions (19) is physically reasonable and normally satisfied. The second condition however can be violated. In this case, the two relaxation models predict different dynamics for the spin polarisation (see Figure (2)B and D). They are inconsistent whatever rates $R_{1, k j}, R_{2, k j}$ we choose. To provide more insight we discuss in the following an instructiv example.

Let us have two nuclei and assume as before that the "effective Hamiltonian" commutes with $H_{Z}$, i.e., has the form (18). Generally, there exists the unique basis, in which the both $H_{0}^{\prime}$ and $H_{Z}$ are diagonal. In terms of Zeeman states (with the first nucleus in the first position, the second nucleus in the second position and the electron separated with the comma), this basis is

$$
\left|\lambda_{1}\right\rangle=|\alpha \alpha, \alpha\rangle, \quad\left|\lambda_{2}\right\rangle=|\beta \beta, \alpha\rangle, \quad\left|\lambda_{3}\right\rangle=|\alpha \alpha, \beta\rangle, \quad\left|\lambda_{4}\right\rangle=|\beta \beta, \beta\rangle,
$$



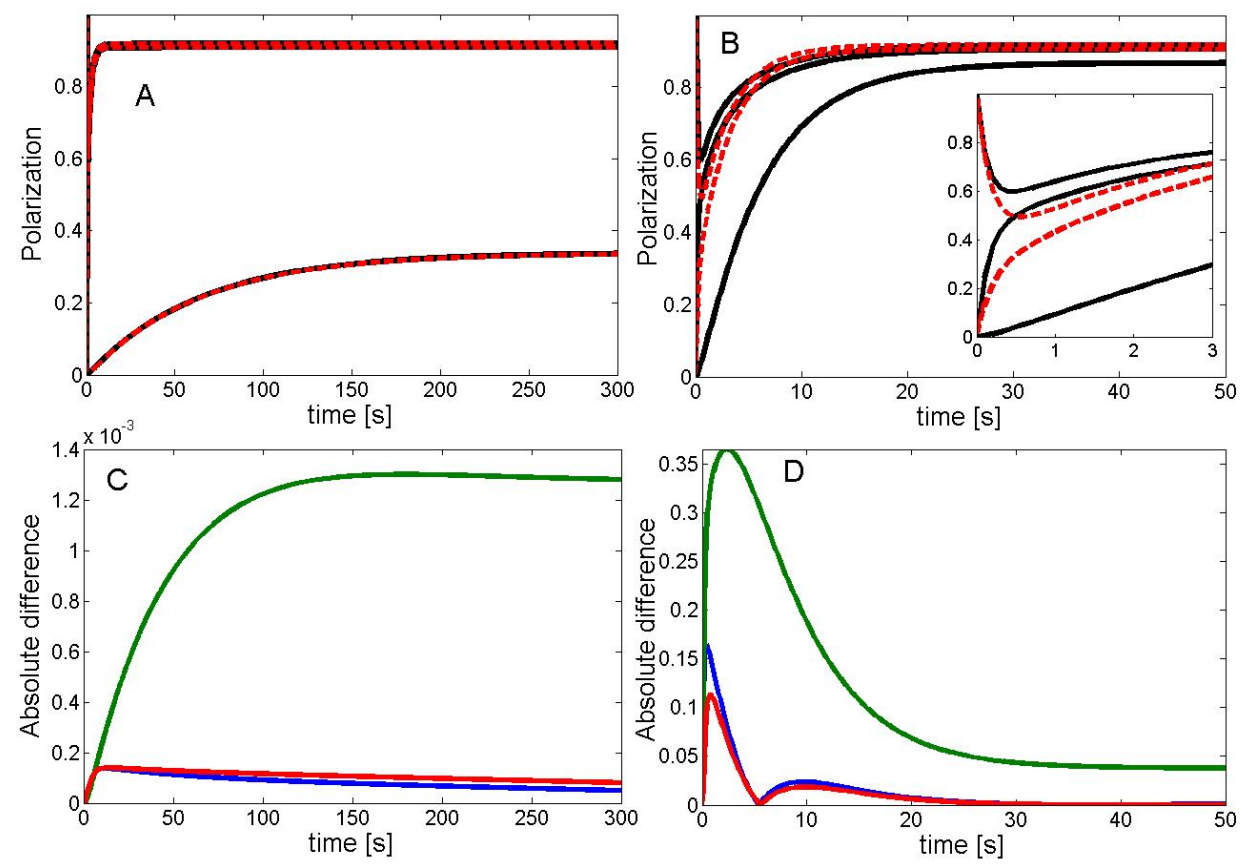

Figure 2. Comparison between the two models (10) (red) and (14) (black). A: Time course of the polarisation of the electron and two nuclear spins. $\omega_{I}=144 \mathrm{MHz}, \mathrm{MW}=0.1 e 6, R_{1 e}=1, R_{2 e}=1 e 5, r_{1 n}=$ $1 e-2, r_{2 n}=1 e 4, A_{0}=[0.155,-0.016] \times 1 e 6, A_{+}=[0.466,0.015] \times 1 e 6, d=64.57 \mathrm{~Hz} . \mathrm{C}:$ shows the difference between the two simulations in A. B: All parameters are the same apart from the interaction strengths $A_{0}=[-0.015,-0.015] \times 1 e 6, A_{+}=[0.43,0.015] \times 1 e 6, d=15 \mathrm{~Hz}, \mathrm{D}$ : shows the difference between the two simulations in B. The maximal absolute error is more the $30 \%$ of the spin polarisation

$$
\begin{gathered}
\left|\lambda_{5}\right\rangle=\cos \phi_{+}|\alpha \beta, \alpha\rangle+\sin \phi_{+}|\beta \alpha, \alpha\rangle, \quad\left|\lambda_{6}\right\rangle=\cos \phi_{+}|\beta \alpha, \alpha\rangle-\sin \phi_{+}|\alpha \beta, \alpha\rangle, \\
\left|\lambda_{7}\right\rangle=\cos \phi_{-}|\alpha \beta, \beta\rangle+\sin \phi_{-}|\beta \alpha, \beta\rangle, \quad\left|\lambda_{8}\right\rangle=\cos \phi_{-}|\beta \alpha, \beta\rangle-\sin \phi_{-}|\alpha \beta, \beta\rangle, \\
\tan \phi_{ \pm}= \pm \frac{1}{d_{12}}\left(\Delta_{A}-\sqrt{\Delta_{A}^{2}+d_{12}^{2}}\right), \quad \Delta_{A}=\frac{A_{1}-A_{2}}{2}
\end{gathered}
$$

The second of conditions (19) is violated when $\left|d_{12}\right| \gg|\Delta|$. In this case, $\tan \phi_{ \pm}=$ F sign $d_{12}$ and the following mixing of Zeeman states occurs (for $d_{12}>0$ )

$$
\begin{aligned}
& \left|\lambda_{5}\right\rangle=\frac{|\alpha \beta, \alpha\rangle-|\beta \alpha, \alpha\rangle}{\sqrt{2}}, \quad\left|\lambda_{6}\right\rangle=\frac{|\beta \alpha, \alpha\rangle+|\alpha \beta, \alpha\rangle}{\sqrt{2}}, \\
& \left|\lambda_{7}\right\rangle=\frac{|\alpha \beta, \beta\rangle+|\beta \alpha, \beta\rangle}{\sqrt{2}}, \quad\left|\lambda_{8}\right\rangle=\frac{|\beta \alpha, \beta\rangle-|\alpha \beta, \beta\rangle}{\sqrt{2}} .
\end{aligned}
$$

We have, for example,

$$
\begin{gathered}
O_{56}=\frac{1}{2}\left(\frac{1}{2}+S_{z}\right)\left(I_{1+} I_{2-}-I_{1-} I_{2+}+I_{1 z}-I_{2 z}\right), \\
\Gamma O_{56}=\frac{1}{2}\left[\left(c_{1}+c_{2} S_{z}\right)\left(I_{1+} I_{2-}-I_{1-} I_{2+}\right)+\left(r_{1}+d_{1} S_{z}\right) I_{1 z}-\left(r_{2}+d_{2} S_{z}\right) I_{2 z}\right],
\end{gathered}
$$




$$
c_{1}=\frac{1}{2} \sum_{k=1}^{2} \frac{1}{T_{2 n, k}}, \quad c_{2}=\frac{1+p_{0}}{T_{1 e}}+2 c_{1}, \quad r_{k}=\frac{1}{2 T_{1 n, k}}, \quad d_{k}=\left(\frac{1+p_{0}}{T_{1 e}}+\frac{1}{T_{1 n, k}}\right) .
$$

Projecting onto $O_{56}$ gives

$$
\hat{\Gamma} O_{56}=R O_{56}+P
$$

where

$$
R=\operatorname{Tr}\left(\hat{\Gamma} O_{56}, O_{65}\right) \sim c_{1},
$$

$O_{56} \perp P=\hat{\Gamma} O_{56}-R O_{56} \sim \frac{c_{1}}{2}\left(\frac{1}{2}+S_{z}\right)\left(I_{1+} I_{2-}-I_{1-} I_{2+}+I_{2 z}-I_{1 z}\right)=-c_{1} O_{65}$.

We see that the projection $R O_{56}$ and the orthogonal component $P$ are of the same order $\sim c_{1}$ which is the average between the transverse rates $1 / T_{2 n, 1}$ and $1 / T_{2 n, 2}$. This means that the action of the relaxation superoperator $\hat{\Gamma}$ on the non-diagonal element $O_{56}$ is strongly not proportional to $O_{56}$. This will be the case for any nondiagonal elements $O_{k j}$ composed of strongly mixed Zeeman states. Using the model based on the choice of the eigenbasis of $H_{0}^{\prime}$, we have always $\hat{\Gamma}_{0} O_{k j}=R_{2, k j} O_{k j}$, $k \neq j$.

Thus, both models are inconsistent if the first of conditions (19) is satisfied while the second one is violated. The reason for this discrepancy is the use of the stationary Hamiltonian $H_{0}$ as a reference frame for the relaxation model (14) that is based on the choice of the eigenbasis of $H_{0}^{\prime}$ and that was described in Section 7. This choice of reference frame lead for the interaction parameters used in the simulation to a violation of condition (9). To demonstrate this we provide a list of the values $\epsilon_{k j, k^{\prime} j^{\prime}}=\left|R_{k j, k^{\prime} j^{\prime}} / \Omega_{k j, k^{\prime} j^{\prime}}\right|$ calculated (see formula (8)) for the simulation shown in Figure (2) in Table 1. Condition (9) which requires $\epsilon_{k j, k^{\prime} j^{\prime}} \ll 1$ is not fulfilled for more than $3.6 \%$ of the 1447 non zero elements (4096 in total). Therefore, the intrinsic assumption made during the derivation of the superoperator in section 7 that non-secular terms can always be neglected in this strategy is not valid and the predictions made by model (14) will deviate from the predictions arising from the random fluctuation model (10). For the core nuclei with much stronger hyperfine interactions the values of $\Omega_{k j, k^{\prime} j^{\prime}}$ are always bigger then the relaxation rate $R_{k j, k^{\prime} j^{\prime}}$ and hence there is a good agreement between the two models. This example emphasizes that particular care must be used to choose the correct interaction frame and to introduce physical meaningful relaxation mechanism.

Figure (3) provides another example of the deviations between the two models. In this case we have assumed a spin chain of 4 nuclear spins and one electron with only the first nuclear spin coupled to the electron and all other nuclear spins interacting through dipolar interaction with each other (similar to Vega's JCP paper).

This further example demostrates that the polarisation dynamics can substantially deviate between the uncorrelated fluctuation model (10) and the model that was built from the eigenbasis of the truncated stationary Hamiltonian $H_{0}^{\prime}$. An analysis of the values for $\epsilon_{k j, k^{\prime} j^{\prime}}$ again shows that condition (9) for the removal of the non-secular terms during averaging the time dependent relaxation superoperator is severely violated. Again a substantial number of $\epsilon_{k j, k^{\prime} j^{\prime}}$ are in the order of $10^{9}$, demonstrating that $H_{0}^{\prime}$ cannot be used to fully remove the non-secular terms in 
Table 1. The first representative values of $\epsilon_{k j, k^{\prime} j^{\prime}}=\frac{\left|R_{k j, k^{\prime} j^{\prime}}\right|}{\Omega_{k j, k^{\prime} j^{\prime}}}$ are provided in descending order for both the simulation of the core nuclei and the simulation of the bulk nuclei shown in Fig.2. Note the huge deviation from the condition $\epsilon_{k j, k^{\prime} j^{\prime}} \ll 1$ which is required for the removal of the non-secular terms during averaging the timedependent relaxation superoperator (see Section 5). This provides the explanation why the model (14) which uses $H_{0}^{\prime}$ as a reference frame substantially deviates from the predictions of the uncorrelated random fluctuation model (10). Only the first 21 value for $\epsilon_{k j, k^{\prime} j^{\prime}}$ of the 1447 non-zero values for the core are shown. (There are another 31 values close to 1$)$.

\begin{tabular}{|l|l|}
\hline \multicolumn{2}{|c|}{$\epsilon_{k j, k^{\prime} j^{\prime}}$} \\
\hline Bulk & Core \\
\hline $1.96608 \mathrm{E}+09$ & $2.12767 \mathrm{E}-05$ \\
\hline $1.96608 \mathrm{E}+09$ & $2.12767 \mathrm{E}-05$ \\
\hline $1.96325 \mathrm{E}+09$ & $2.12767 \mathrm{E}-05$ \\
\hline $1.96325 \mathrm{E}+09$ & $2.12767 \mathrm{E}-05$ \\
\hline $1.80224 \mathrm{E}+09$ & $2.12767 \mathrm{E}-05$ \\
\hline $1.80224 \mathrm{E}+09$ & $2.12767 \mathrm{E}-05$ \\
\hline $1.80224 \mathrm{E}+09$ & $2.12767 \mathrm{E}-05$ \\
\hline $1.80224 \mathrm{E}+09$ & $2.12767 \mathrm{E}-05$ \\
\hline $1.80224 \mathrm{E}+09$ & $2.12757 \mathrm{E}-05$ \\
\hline $1.80224 \mathrm{E}+09$ & $2.12757 \mathrm{E}-05$ \\
\hline $1.80224 \mathrm{E}+09$ & $2.12757 \mathrm{E}-05$ \\
\hline $1.80224 \mathrm{E}+09$ & $2.12757 \mathrm{E}-05$ \\
\hline $1.64123 \mathrm{E}+09$ & $2.12757 \mathrm{E}-05$ \\
\hline $1.64123 \mathrm{E}+09$ & $2.12757 \mathrm{E}-05$ \\
\hline $1.63840 \mathrm{E}+09$ & $2.12757 \mathrm{E}-05$ \\
\hline $1.63840 \mathrm{E}+09$ & $2.12757 \mathrm{E}-05$ \\
\hline $8.21487 \mathrm{E}+02$ & $2.12757 \mathrm{E}-05$ \\
\hline $8.21487 \mathrm{E}+02$ & $2.12757 \mathrm{E}-05$ \\
\hline $8.21009 \mathrm{E}+02$ & $2.12757 \mathrm{E}-05$ \\
\hline $8.21009 \mathrm{E}+02$ & $2.12757 \mathrm{E}-05$ \\
\hline $9.08939 \mathrm{E}-01$ & $2.12757 \mathrm{E}-05$ \\
\hline
\end{tabular}

a relaxation model. We can conclude that the use of $H_{0}^{\prime}$ frame can lead to spin dynamic predictions that cannot be explained by spin relaxation arising from a physically reasonable fluctuation model. The relaxation model derived in section 7 (which is equivalent to the one used in Vega's paper) can only be used for the special situation when condition (19) is satisfied.

\section{Extension to more complex relaxation models}

In this section we extent the discussion to more complex relaxation mechanism. Three examples are given based on the fluctuations approach described in section 5. The examples illustrate the typical mechanisms of relaxation in solids and are applicable to the electron-nuclear spin system in a SE DNP model: the electronic relaxation caused by the $g$-anisotropy, the nuclear relaxation caused by the electron as a paramagnetic centre and the electron-nuclear relaxation caused by vibrations of the crystalline lattice near the electronic spin.

\subsection{Electron relaxation caused by g-anisotropy}

The Zeeman interaction of the electronic spin $S$ with the static field $B_{0}$ is mediated by the $g$-tensor, $B_{0} . g . S$. The tensor $g$ depends on the surroundings of the electron 

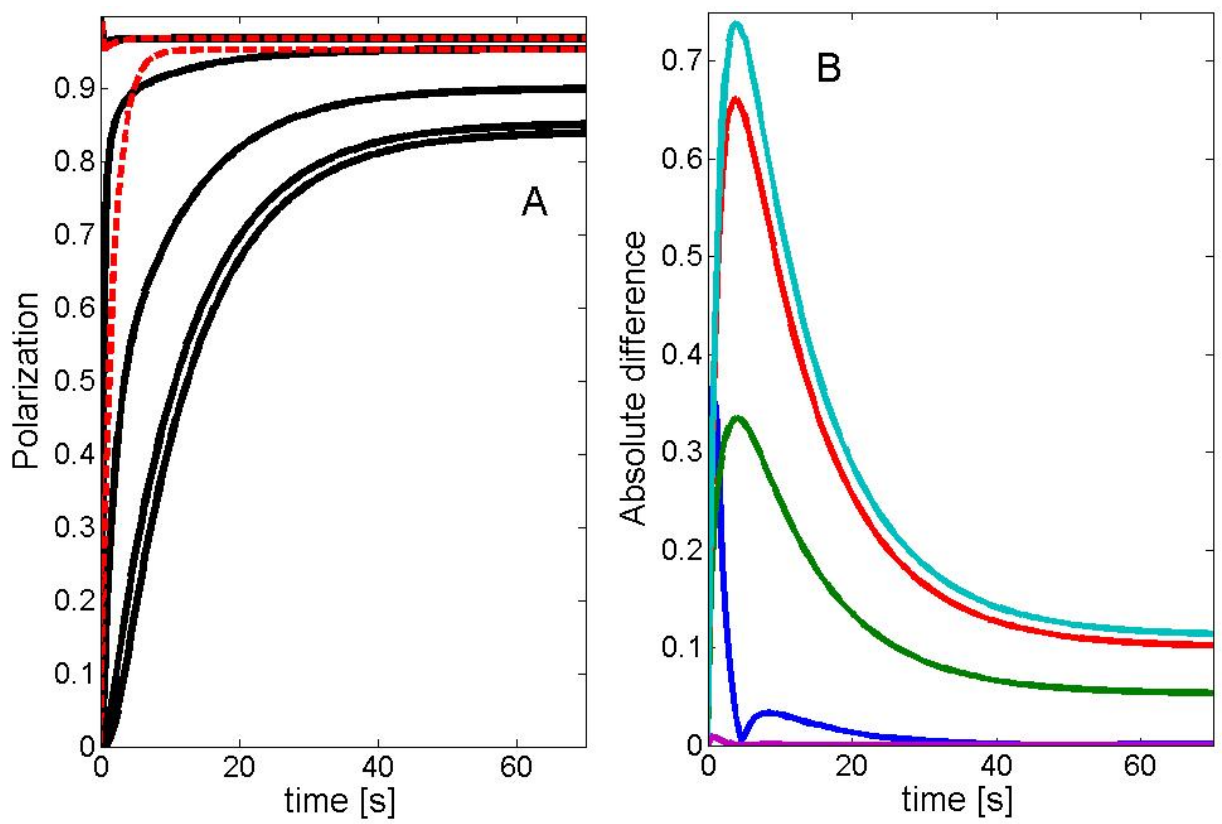

Figure 3. A: Polarisation dynamics in a spin chain consisting of one electron and four nuclear spins, simulated using the relaxation model (14) (red) and (10) (black). B: Difference between the predictions of the two models: blue 1st spin, green 2nd spin, red 3rd spin, light blue 4th spin, magenta electron. Note the huge difference for the prediction of the polarisation of the 3rd and 4th spin. Simulation parameters: $\omega_{I}=36 \mathrm{MHz}, \mathrm{MW}=0.2 e 6, R_{1 e}=1 e 2, R_{2 e}=1 e 5, r_{1 n}=1 e-2, r_{2 n}=1 e 3, A_{0}=[0,0,0,0] \times 1 e 6$, $A_{+}=[0.04,0,0,0] \times 1 e 6, d(1,2)=7, d(2,3)=6, d(3,4)=8.5 \mathrm{~Hz}$.

and is generally anisotropic, i.e., not represented by a simple scalar product between the vectors $B_{0}$ and $S$. In sufficiently symmetric environment, the $g$-anisotropy is relatively small, but can be appreciable in non-symmetric cases.

Considering an ensemble of electronic spins $\left\{S_{k}\right\}$, we have to assume that each electron in the ensemble has its own $g$-tensor $g_{k}$, even when the anisotropic parts of them are small. In this case,

$$
g_{k}=g_{0} \cdot 1+g_{k, a}, \quad\left\|g_{k, a}\right\| \ll\left|g_{0}\right|
$$

Statistically the electronic ensemble is well represented by a single electron $S$ in such way that the Zeeman interaction becomes

$$
H_{Z}=\omega_{S} S_{z}+B_{0} . g_{a} . S, \quad \omega_{S}=g_{0}\left|B_{0}\right|,
$$

where $g_{a}$ is a random spatially distributed tensor. Using the ergodicity principle, we can assume that the tensor $g_{a}=g_{a}(t)$ is a random function of time, interpreting this as that anisotropies of different electron spins have different effective times, which are randomly distributed between them. An analogous assumption is made in liquid state NMR when we regard the spatially distributed ensemble molecular motion as a random temporal motion of a single molecule.

Thus, we can write

$$
H_{Z}=\omega_{S} S_{z}+H_{f}(t), \quad H_{f}(t)=f_{z}(t) S_{z}+f_{+}(t) S_{+}+f_{-}(t) S_{-}
$$

with random scalar functions $f_{\beta}(t), \beta=z, \pm$. We can assume that $f_{\beta}(t)$ are random stationary processes with zero ensemble averages and some correlations functions

$$
\overline{f_{\beta}(t)}=0, \quad g_{\alpha \beta}(\tau)=\overline{f_{\alpha}(t) f_{\beta}^{*}(t-\tau)},
$$


so we follow the fluctuations approach described in section 5 .

The operators $S_{\beta}, \beta=z, \pm$ are eigenvectors of the superoperator $\hat{H}_{Z}$ with the eigenoperator $0, \pm \omega_{S}$ respectively. This leads to the straightforward formula

$$
\hat{\Gamma}_{S}=R_{2} \hat{S}_{z}+R_{1}\left(\hat{S}_{+} \hat{S}_{-}+\hat{S}_{-} \hat{S}_{+}\right)
$$

with

$$
R_{2}=\frac{1}{2} \int_{-\infty}^{+\infty} g_{z z}(\tau) d \tau, \quad R_{1}=\frac{1}{2} \int_{-\infty}^{+\infty} g_{++}(\tau) e^{i \omega_{S} \tau} d \tau=\frac{1}{2} \int_{-\infty}^{+\infty} g_{--}(\tau) e^{i \omega_{S} \tau} d \tau
$$

\subsection{Nuclear relaxation caused by electron as paramagnetic centre}

To describe the electron-nuclear hyperfine interaction in a dielectric solids between a radical centre with a locally confine electron and the surrounding nuclei we can focus mainly on the dipolar interaction and ignore the Fermi contact interaction. Taking into account the huge electronic Larmor frequency $\omega_{S}$ in the Zeeman frame we can write the hyperfine interaction in the reduced form

$$
H_{I S}=V S_{z}, \quad V=\sum_{k}\left(2 A_{k 0} I_{k z}+A_{k+} I_{k+}+A_{k-} I_{k-}\right)
$$

The role of the electron as a paramagnetic centre (or impurity) is described as follows (see Bloemebrgen's spin diffusion, Abragam-Goldman's relaxation of II kind and others).

It is assumed that during the evolution, a spontaneous exchange occurs between the subspaces of the "up" and "down" states of the electronic spin. Due to the presence of the terms with $I_{k \pm} S_{z}$ in $H_{I S}$, this random process affects the nuclear spins in the form of a random field seen by them via spontaneous changes of the sign of the coefficients $A_{k \pm}$. These fluctuations are seen simultaneously by all nuclei grouped to the corresponding hyperfine interaction terms. The typical correlation time is comparable with the electronic longitudinal time $T_{1 e}$, so can lead to processes with longer times than $T_{1 e}$. However, this can be appreciable and crucial for the nuclei in close electron vicinity.

In terms of fluctuations, this gives

$$
H_{f}(t)=f(t) V_{+}+f^{*}(t) V_{-}, \quad V_{ \pm}=\sum_{k} A_{k \pm} I_{k \pm}
$$

where $f(t)$ is a random scalar function of time. We can assume again that it describes a stationary process with zero average and some correlation function

$$
\overline{f(t)}=0, \quad g(\tau)=\overline{f(t) f^{*}(t-\tau)} .
$$

The operataors $V_{ \pm}$are eigenvectors of $\hat{H}_{Z}$ with the eigenvalues $\pm \omega_{I}$ respectively. Using the fluctuations approach, this leads to the following superoperator

$$
\hat{\Gamma}_{I}=\tau_{0}\left(\hat{V}_{+} \hat{V}_{-}+\hat{V}_{-} \hat{V}_{+}\right), \quad \tau_{0}=\frac{1}{2} \int_{-\infty}^{+\infty} g(\tau) e^{i \omega_{I} \tau} d \tau
$$


The simplest realization

$$
g(\tau)=g(-\tau)=\frac{1}{4} \exp \left(-\frac{|\tau|}{T_{1 e}}\right)
$$

leads to the formula

$$
\tau_{0}=\frac{1}{4} \frac{T_{1 e}}{1+\omega_{I}^{2} T_{1 e}^{2}} \sim \frac{1}{4 \omega_{I}^{2}} \frac{1}{T_{1 e}}
$$

It is seen that the relaxation rates are $\sim \epsilon_{k} / T_{1 e}$ with $\epsilon_{k}=\left|A_{k \pm} / \omega_{I}\right|^{2}$, so they tend to be larger for nuclei closer to the electron and smaller for remote nuclei.

\subsection{Electron-nuclear relaxation via vibrations of crystalline lattice near electron}

The electron-nuclear interaction can lead to another mechanism connected with vibrations of the crystalline lattice in the electron vicinity, as follows.

Even at low temperatures, the position of the electronic spin (unlike the nuclear spins) is not fixed in space, it is found randomly in time in some volume near its average position. This causes vibrations of the crystalline lattice near the electron in the form of the phonon lattice sound. This random noise is seen by the nuclear spins via fluctuations of the coefficients of the hyperfine interaction which depend on orientations of the electron-nuclear pairs and so depend on the random electron position. In the Zeeman frame with large Larmor frequencies $\omega_{I, S}$, the dominating part of the electron-nuclear interaction in the purely Zeeman part

$$
H_{I S, Z}=2 \sum_{k} A_{k, 0} I_{k z} S_{z}
$$

The fluctuations of the coefficients $A_{k, 0}$ caused by the above vibrations can be written as

$$
H_{f}(t)=\sum_{k} f_{k}(t) V_{k}, \quad V_{k}=2 A_{k, 0} I_{k z} S_{z}
$$

where $f_{k}(t)$ are real scalar functions of time describing random stationary processes with zero averages and some correlation functions

$$
\overline{f_{k}(t)}=0, \quad g_{k j}(\tau)=\overline{f_{k}(t) f_{j}(t-\tau)} .
$$

The operators $V_{k}$ belong to the zero eigenspace of $\hat{H}_{Z}$. This leads to the superoperator

$$
\hat{\Gamma}_{I S}=\sum_{k, j} \tau_{k j} \hat{V}_{k} \hat{V}_{j}, \quad \tau_{k j}=\frac{1}{2} \int_{-\infty}^{+\infty} g_{k j}(\tau) d \tau .
$$

It is seen that $\hat{\Gamma}_{I S}$ affects only non-Zeeman operators with rates proportional to $A_{k, 0} A_{j, 0}$.

Combining the models (21), (22), (23) and applying the thermalization, we obtain

$$
\dot{\sigma}=-i \hat{H} \sigma-\hat{\Gamma} \sigma
$$




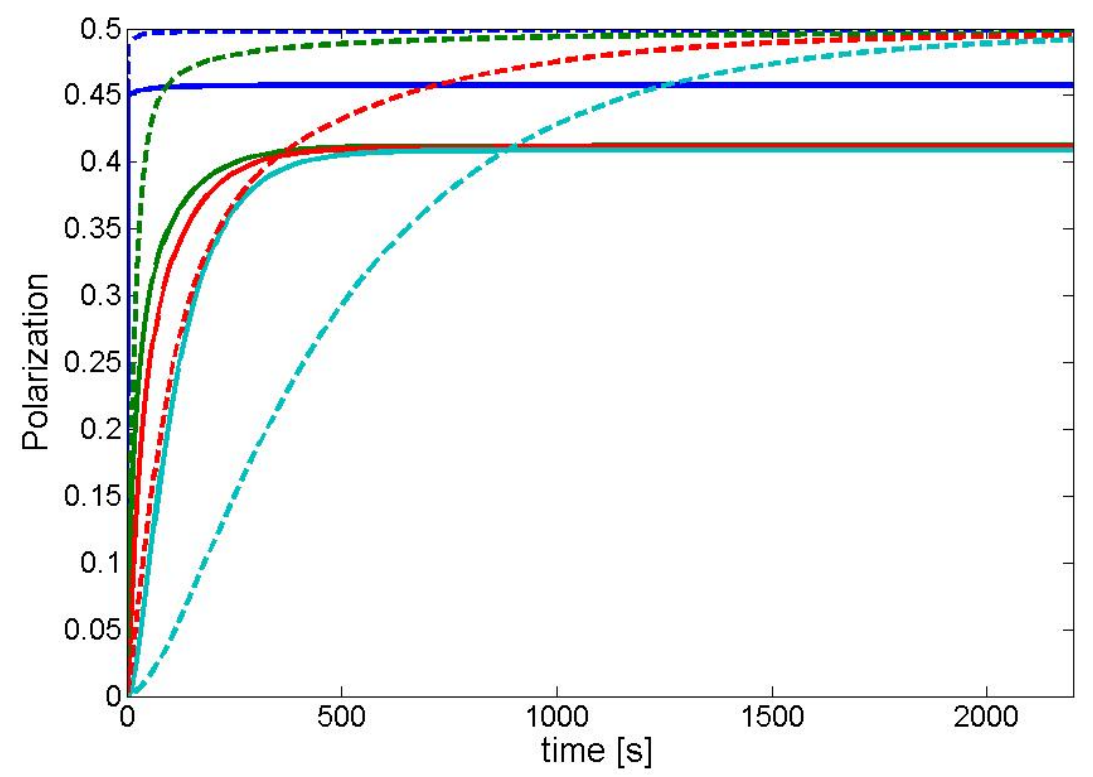

Figure 4. Comparison of the nuclear polarisation dynamics for four ${ }^{13} \mathrm{C}$ nuclei calculated using the simple uncorrelated random fluctuation model (10) (solid line) and a model taking all other mechanisms into account (broken line). Simulation parameters were $\mathrm{MW}=0.1 \mathrm{MHz}, R_{1 e}=1 e 0, R_{2 e}=1 e 5, r_{1 n}=6.5 e-$ $4, r_{2 n}=1 e 4$, for the random fluctuation model and $R_{1 e}=4, R_{2 e}=1.12 e 5, r_{1 n}=(6.55 e-4,8.83 e-$ $06,6.40 e-07,4.13 e-08), r_{2 n}=(9527,1283,930,600)$ for the more comprehensive model. Note that for the comprehensive model all nuclear spins reach eventually the same polarisation level

with

$$
\begin{gathered}
\hat{\Gamma} \sigma=\hat{\Gamma}_{S} \sigma+\hat{\Gamma}_{I} \sigma+\hat{\Gamma}_{I S} \sigma+\hat{\Gamma}_{t h}, \\
\hat{\Gamma}_{t h} \sigma=2 p_{0} R_{1}\left(S_{+} \sigma S_{-}-S_{-} \sigma S_{+}+S_{z} \sigma+\sigma S_{z}\right) .
\end{gathered}
$$

To find connections between $R_{1,2}, \tau_{0}, \tau_{k j}$ and the effective transverse and longitudinal relaxation times, we can calculate according to (24)

$$
\begin{gathered}
\hat{\Gamma} S_{z}=4 R_{1} S_{z}, \quad \hat{\Gamma} S_{ \pm}=\left(R_{2}+2 R_{1}+\sum_{k} \tau_{k k}\left|A_{k 0}\right|^{2}+\sum_{k \neq j} \tau_{k j} A_{k 0} A_{j 0} I_{k z} I_{j z}\right) S_{ \pm} \\
\hat{\Gamma} I_{k z}=4 \tau_{0}\left|A_{k \pm}\right|^{2} I_{k z}+8 p_{0} R_{1} I_{k z} S_{z} \\
\hat{\Gamma} I_{k \pm}=\left(2 \tau_{0}\left|A_{k \pm}\right|^{2}+\tau_{k k}\left|A_{k 0}\right|^{2}\right) I_{k \pm}+8 p_{0} R_{1} I_{k \pm} S_{z}
\end{gathered}
$$

getting effectively

$$
\begin{gathered}
\frac{1}{T_{1 e}}=4 R_{1}, \quad \frac{1}{T_{2 e}}=R_{2}+2 R_{1}+\sum_{k} \tau_{k k}\left|A_{k 0}\right|^{2}, \\
\frac{1}{T_{1 n, k}}=4 \tau_{0}\left|A_{k \pm}\right|^{2}, \quad \frac{1}{T_{2 n, k}}=2 \tau_{0}\left|A_{k \pm}\right|^{2}+\tau_{k k}\left|A_{k 0}\right|^{2} .
\end{gathered}
$$




\section{Conclusions and Outlook}

The polarisation enhancement of the nuclear spin ensemble by solid effect DNP is the result of both the irradiation of one of the electron satellite frequencies $\left(\omega_{S} \pm \omega_{I}\right)$ and the relaxation processes in response to this preturbation. Relaxation needs to be incorporated into a quantum mechancial model to provide predictions that can be used for comparison with experimental data. We reviewed and compared several strategies and pointed out under which conditions these strategies provide predictions in close agreement and discussed under which conditions they fail to agree. In particular we outlined our strategy to incorporate relaxation through the assumption of fluctuations that modulate the interaction of the electrons and the nuclear spins with their respective environment. The mathematical advantage of our strategy is the avoidance of the diagnalisations of large matrices which enables us to increase the number of coupled nuclear spins in the quantum system. Since the dynamics of the nuclear polarisation depends on the number of coupled nuclear spins in the spin system it is important to maximise this number in model simulations to obtain predictions close to the experimental observations.

\section{Appendix}

\subsection{Invariance of the relaxation superoperator in the Lindblad-Kossakowski form}

After proceeding to the interaction frame by the rule (4), the relaxation superoperator (3) is transformed to

$$
-\hat{\Gamma}^{\prime} \bar{\sigma}=\sum_{k=1}^{N^{2}-1} \gamma_{k}\left[\bar{L}_{k} \bar{\sigma} \bar{L}_{k}^{*}-\frac{1}{2}\left(\bar{\sigma} \bar{L}_{k}^{*} \bar{L}_{k}+\bar{L}_{k}^{*} \bar{L}_{k} \bar{\sigma}\right)\right], \quad \bar{L}_{k}=e^{i \hat{H}_{z} t} L_{k} .
$$

The Liouville space admits the following expansion into eigenspaces $V_{p q}$ of the superoperator $\hat{H}_{Z}$ ( $n$ is the number of nuclei $)^{1}$

$$
\mathcal{L}=\sum_{p=-n}^{n} \sum_{q=-1}^{1} V_{p q}, \quad \hat{H}_{Z} v=\Omega_{p q} v, \quad \Omega_{p q}=p \omega_{I}+q \omega_{S}, \quad v \in V_{p q} .
$$

Using the expansions

$$
L_{k}=\sum_{p, q} a_{k, p q} l_{k, p q}, \quad l_{k, p q} \in V_{p q}, \quad \bar{L}_{k}=\sum_{p, q} a_{k, p q} e^{i \Omega_{p q} t} l_{k, p q}
$$

we obtain

$$
\begin{gathered}
\bar{L}_{k} \bar{\sigma} \bar{L}_{k}^{*}-\frac{1}{2}\left(\bar{\sigma} \bar{L}_{k}^{*} \bar{L}_{k}+\bar{L}_{k}^{*} \bar{L}_{k} \bar{\sigma}\right)= \\
=\sum_{p, q, p^{\prime}, q^{\prime}} e^{i\left(\Omega_{p q}-\Omega_{p^{\prime} q^{\prime}}\right) t} a_{k, p q} a_{k, p^{\prime} q^{\prime}}^{*}\left[l_{k, p q} \bar{\sigma} l_{k, p^{\prime} q^{\prime}}^{*}-\frac{1}{2}\left(\bar{\sigma} l_{k, p^{\prime} q^{\prime}}^{*} l_{k, p q}+l_{k, p^{\prime} q^{\prime}}^{*} l_{k, p q} \bar{\sigma}\right)\right] .
\end{gathered}
$$

\footnotetext{
${ }^{1}$ We assume that the magnitudes $\Omega_{p q}$ are all well distinguished, which is true for protons at high field. This is true also for nuclei with smaller gyromagnetic ratios because in this case $n<\left|\omega_{S} / \omega_{I}\right|$.
} 
Effectively, the non-secular terms are averaged out, so $\hat{\Gamma}^{\prime}$ should be timeindependent. This means

$$
a_{k, p q} a_{k, p^{\prime} q^{\prime}}^{*}=0, \quad(p q) \neq\left(p^{\prime} q^{\prime}\right)
$$

so each $L_{k}$ belongs completely to one and only one of the eigenspaces $V_{p q}$, i.e., each $L_{k}$ is an eigenvector of the Zeeman superoperator $\hat{H}_{Z}$.

Thus, we conclude that the effective relaxation superoperator (3) in the interaction frame is such that the set $\left\{L_{k}\right\}$ of Lindblad-Kossakowski operators is an orthogonal set of traceless eigenvectors of the superoperator $\hat{H}_{Z}$.

\subsection{Derivation of the relaxation superoperator in the \\ Lindblad-Kossakowski form starting from a fluctuation model}

We can assume that the fluctuation $H_{f}$ is built of a full set $\left\{F_{p q, m}\right\}$ of orthonormal eigenoperators of $\hat{H}_{Z}$. In the case that some eigenoperators are absent, we assign the zero value to the corresponding coefficient. Taking into account the multiplicities of eigenvalues, $p \in \overline{-n, n}, q \in \overline{-1,1}, m \in \overline{1, \operatorname{dim} V_{p q}}$.

We have

$$
\begin{gathered}
\forall m \quad \hat{H}_{Z} F_{p q, m}=\Omega_{p q} F_{p q, m}, \quad \Omega_{p q}=p \omega_{I}+q \Omega_{S}, \\
F_{p q, m}^{\prime} \equiv e^{i \hat{H}_{Z} t} F_{p q, m}=e^{i \Omega_{p, q} t} F_{p q, m} .
\end{gathered}
$$

Hence,

$$
\begin{gathered}
H_{f}^{\prime}(t)=\sum_{p, q} \sum_{m} f_{p q, m}(t) F_{p q, m}^{\prime}(t)=\sum_{p, q} \sum_{m} f_{p q, m}(t) e^{i \Omega_{p, q} t} F_{p q, m}, \\
\hat{H}_{f}^{\prime}(t) \hat{H}_{f}^{\prime}(t-\tau)=\hat{H}_{f}^{\prime}(t) \hat{H}_{f}^{\prime *}(t-\tau)= \\
=\sum_{p, q, p^{\prime}, q^{\prime}} \sum_{m, m^{\prime}} f_{p q, m}(t) f_{p^{\prime} q^{\prime}, m^{\prime}}^{*}(t-\tau) \exp \left[i \Omega_{p q} t-i \Omega_{p^{\prime} q^{\prime}}(t-\tau)\right] \hat{F}_{p q, m} \hat{F}_{p^{\prime} q^{\prime}, m^{\prime}}^{*}
\end{gathered}
$$

where^ over spin operators denotes the commutation superoperator of these operators. Taking the ensemble average and truncating non-secular terms, we obtain

$$
\begin{gathered}
\overline{\hat{H}_{f}^{\prime}(t) \hat{H}_{f}^{\prime}(t-\tau)}=\sum_{p, q} \sum_{m, m^{\prime}} c_{p q, m m^{\prime}}(\tau) \exp \left(i \Omega_{p q} \tau\right) \hat{F}_{p q, m} \hat{F}_{p q, m^{\prime}}^{*}, \\
c_{p q, m m^{\prime}}=\overline{f_{p q, m}(t) f_{p q, m^{\prime}}^{*}(t-\tau)} .
\end{gathered}
$$

This leads to the general formula

$$
\hat{\Gamma}=\sum_{p, q} \sum_{m, m^{\prime}} C_{p q, m m^{\prime}} \hat{F}_{p q, m} \hat{F}_{p q, m^{\prime}}^{*}, \quad C_{p q, m m^{\prime}}=\frac{1}{2} \int_{-\infty}^{+\infty} c_{p q, m m^{\prime}}(\tau) \exp \left(i \Omega_{p q} \tau\right) d \tau .
$$




\section{Thermalization and reduction to Lindblad-Kossakowski form}

It is seen that the form (27) is generally not in the Lindblad-Kossakowski form. The thermal correction is needed as well as the reduction of the doublecommutators in a proper way. This is done as follows.

Taking Hermitian conjugate and permuting indices, we obtain

$$
\hat{\Gamma}=\sum_{p, q} \sum_{m, m^{\prime}} C_{p q, m m^{\prime}} \hat{F}_{p q, m} \hat{F}_{p q, m^{\prime}}^{*}=\sum_{p, q} \sum_{m, m^{\prime}} C_{p q, m^{\prime} m}^{*} \hat{F}_{p q, m^{\prime}}^{*} \hat{F}_{p q, m}
$$

Since

$$
C_{p q, m^{\prime} m}^{*}=C_{p q, m m^{\prime}}
$$

this gives

$$
\hat{\Gamma}=\frac{1}{2} \sum_{p, q} \sum_{m, m^{\prime}} C_{p q, m m^{\prime}}\left(\hat{F}_{p q, m} \hat{F}_{p q, m^{\prime}}^{*}+\hat{F}_{p q, m^{\prime}}^{*} \hat{F}_{p q, m}\right)
$$

Consider the two superoperators

$$
\begin{aligned}
& \hat{U}_{p q, m m^{\prime}}^{+} \sigma=F_{p q, m} \sigma F_{p q, m^{\prime}}^{*}-\frac{1}{2}\left(\sigma F_{p q, m^{\prime}}^{*} F_{p q, m}+F_{p q, m^{\prime}}^{*} F_{p q, m} \sigma\right) \\
& \hat{U}_{p q, m m^{\prime}}^{-} \sigma=F_{p q, m^{\prime}}^{*} \sigma F_{p q, m}-\frac{1}{2}\left(\sigma F_{p q, m} F_{p q, m^{\prime}}^{*}+F_{p q, m} F_{p q, m^{\prime}}^{*} \sigma\right)
\end{aligned}
$$

and rewrite $\hat{\Gamma}$ as

$-\hat{\Gamma}^{\prime}=\sum_{p, q} \sum_{m, m^{\prime}} C_{p q, m m^{\prime}}\left(\hat{U}_{p q, m m^{\prime}}^{+}+\hat{U}_{p q, m m^{\prime}}^{-}\right)+\sum_{p, q} \sum_{m, m^{\prime}} C_{p q, m m^{\prime}}^{-}\left(\hat{U}_{p q, m m^{\prime}}^{+}-\hat{U}_{p q, m m^{\prime}}^{-}\right)$.

In accordance with the formula

$$
\hat{F}_{p q, m} \hat{F}_{p q, m^{\prime}}^{*}+\hat{F}_{p q, m^{\prime}}^{*} \hat{F}_{p q, m}=-2\left(\hat{U}_{p q, m m^{\prime}}^{+}+\hat{U}_{p q, m m^{\prime}}^{-}\right),
$$

the superoperator $\hat{\Gamma}^{\prime}$ is in the Lindblad-Kossakowski form and coincides with $\hat{\Gamma}$ for $C_{p q, m m^{\prime}}^{-}=0$. We can choose $C_{p q, m m^{\prime}}^{-}$in such way that

$$
\hat{\Gamma}^{\prime} \sigma_{t h}=0
$$

Then the master equation

$$
\dot{\sigma}=-i \hat{H}_{0} \sigma-\hat{\Gamma}^{\prime} \sigma
$$

is the needed homogeneous Lindblad-Kossakowski form where the full density operator should be used. Here we can apply the approximation

$$
\sigma_{t h}=\frac{1}{N}\left(1-2 p_{0} S_{z}\right), \quad p_{0}=\tanh \frac{\hbar \Omega_{S}}{2 k_{B} T} .
$$


11.3. Derivation of the relaxation superoperator based on the uncorrelated random fluctuation model

Let us introduce the superoperators

$$
\hat{U}_{z} \sigma=S_{z} \sigma S_{z}-\frac{1}{2}\left(\sigma S_{z} S_{z}+S_{z} S_{z} \sigma\right), \quad \hat{U}_{ \pm} \sigma=S_{ \pm} \sigma S_{\mp}-\frac{1}{2}\left(\sigma S_{\mp} S_{ \pm}+S_{\mp} S_{ \pm} \sigma\right),
$$

$\hat{u}_{k z} \sigma=I_{k z} \sigma I_{k z}-\frac{1}{2}\left(\sigma I_{k z} I_{k z}+I_{k z} I_{k z} \sigma\right), \quad \hat{u}_{k \pm} \sigma=I_{k \pm} \sigma I_{k \mp}-\frac{1}{2}\left(\sigma I_{k \mp} I_{k \pm}+I_{k \mp} I_{k \pm} \sigma\right)$

and the commutation superoperators

$$
\hat{L} \equiv[L, \cdot], \quad L=S_{z}, S_{ \pm}, I_{s z}, I_{s \pm} .
$$

Due to the relations valid for any spin $1 / 2$

$$
I_{z}^{2}=\frac{1}{4}, \quad I_{ \pm} I_{\mp}=\frac{1}{2} \pm I_{z}
$$

we have

$$
\begin{gathered}
\hat{U}_{z}=-\frac{1}{2} \hat{S}_{z}^{2}, \quad \hat{u}_{k z}=-\frac{1}{2} \hat{I}_{k z}^{2}, \\
\hat{U}_{+}+\hat{U}_{-}=-\frac{1}{2}\left(\hat{S}_{+} \hat{S}_{-}+\hat{S}_{-} \hat{S}_{+}\right), \quad \hat{u}_{k+}+\hat{u}_{k-}=-\frac{1}{2}\left(\hat{I}_{k+} \hat{I}_{k-}+\hat{I}_{k-} \hat{I}_{k+}\right), \\
\left(\hat{U}_{+}-\hat{U}_{-}\right) \sigma=S_{+} \sigma S_{-}-S_{-} \sigma S_{+}+\sigma S_{z}+S_{z} \sigma, \\
\left(\hat{u}_{k+}-\hat{u}_{k-}\right) \sigma=I_{k+} \sigma I_{k-}-I_{k-} \sigma I_{k+}+\sigma I_{k z}+I_{k z} \sigma .
\end{gathered}
$$

This gives

$$
\begin{gathered}
\hat{\Gamma} \sigma=R_{2} \hat{S}_{z}^{2} \sigma+R_{1}\left(\hat{S}_{+} \hat{S}_{-}+\hat{S}_{-} \hat{S}_{+}\right) \sigma+R_{3}\left(S_{+} \sigma S_{-}-S_{-} \sigma S_{+}+\sigma S_{z}+S_{z} \sigma\right)+ \\
+\sum_{k=1}^{n}\left[r_{2 k} \hat{I}_{k z}^{2} \sigma+r_{1 k}\left(\hat{I}_{k+} \hat{I}_{k-}+\hat{I}_{k-} \hat{I}_{k+}\right) \sigma+r_{3 k}\left(I_{k+} \sigma I_{k-}-I_{k-} \sigma I_{k+}+\sigma I_{k z}+I_{k z} \sigma\right)\right]
\end{gathered}
$$

where $R_{j}, r_{j k}$ are some rates to be specified.

11.4. Comparison of the action of the two models $\Gamma_{0}$ and $\Gamma$ for core nuclei

In this basis, each non-diagonal element $O_{k j}=v_{k} v_{j}^{*}, k \neq j$, is represented by one of the following forms

$$
O_{k j}=S_{\beta} O_{Z} \prod_{s=1}^{m} I_{s \beta_{s}}, \quad O_{k j}=\left(\frac{1}{2} \pm S_{z}\right) O_{Z} \prod_{s=1}^{m} I_{s \beta_{s}}, \quad \beta, \beta_{s}= \pm, \quad m \geq 1
$$


where $O_{Z}$ is a combination of Zeeman orders built of spins other than $S, I_{s}$. For example (the electronic state is separated with the comma),

$$
|\alpha \beta, \alpha\rangle\left\langle\beta \beta, \beta\left|=S_{+} I_{1+}\left(\frac{1}{2}-I_{2 z}\right), \quad\right| \alpha \beta, \alpha\right\rangle\langle\beta \beta, \alpha|=\left(\frac{1}{2}+S_{z}\right) I_{1+}\left(\frac{1}{2}-I_{2 z}\right) .
$$

Since $T_{2 e}, T_{2 n, k} \ll T_{1 e}, T_{1 n, k}$, the acton of our relaxation superoperator $\hat{\Gamma}$ on $O_{k j}$ in the first case is well approximated as

$$
\hat{\Gamma} O_{k j}=R_{2, k j}^{\prime} O_{k j}, \quad R_{2, k j}^{\prime}=\frac{1}{T_{2 e}}+\sum_{s=1}^{m} \frac{1}{T_{2 n, s}} .
$$

In the second case,

$$
\hat{\Gamma} O_{k j} \sim\left(\frac{1}{2} \sum_{s=1}^{m} \frac{1}{T_{2 n, s}}\right) O_{Z} \prod I_{s \beta_{s}}+\left(\frac{p_{0} \pm 1}{T_{1 e}} \pm \sum_{s=1}^{m} \frac{1}{T_{2 n, s}}\right) O_{Z} S_{z} \prod I_{s \beta_{s}}
$$

which leads to the approximation

$$
\hat{\Gamma} O_{k j}=R_{2, k j}^{\prime \prime} O_{k j}, \quad R_{2, k j}^{\prime \prime}=\sum_{s=1}^{m} \frac{1}{T_{2 n, s}} .
$$

This means that the result of action of $\hat{\Gamma}$ on $O_{k j}$ is approximately proportional to $O_{k j}$. Hence, in the model $\hat{\Gamma}_{0}$, we should let

$$
\hat{\Gamma}_{0} O_{k j}=R_{2, k j} O_{k j}, \quad R_{2, k j}=\operatorname{Tr}\left(\left(\hat{\Gamma} O_{k j}\right) O_{j k}\right) .
$$

In this case, the "transverse parts" of the both relaxation superoperators will be close.

Each diagonal element is represented as

$$
O_{k k}=\left(\frac{1}{2}+\beta S_{z}\right) \prod_{s=1}^{n}\left(\frac{1}{2}+\beta_{s} I_{s z}\right), \quad \beta, \beta_{s}= \pm
$$

For example,

$$
|\alpha \beta, \alpha\rangle\langle\alpha \beta, \alpha|=\left(\frac{1}{2}+S_{z}\right)\left(\frac{1}{2}+I_{1 z}\right)\left(\frac{1}{2}-I_{2 z}\right)
$$

The action of the relaxation superoperator $\hat{\Gamma}$ on $O_{k k}$ gives a traceless combination of Zeeman orders. Any such combination is expanded into a combination of operators $O_{k k}-O_{j j}$ exactly as in the model $\hat{\Gamma}_{0}$. Hence, we should choose

$$
\hat{\Gamma}_{0} O_{k k}=\sum_{j \neq k} R_{1, k j}\left(O_{k k}-O_{j j}\right), \quad R_{1, k j}=-\operatorname{Tr}\left(\left(\hat{\Gamma} O_{k k}\right) O_{j j}\right)
$$

to get the both "longitudinal parts" close to each other. 


\subsection{Numerical implementation of the Lindblad-Kossakowski relaxation superoperator form}

In this section we recapitulate some of the information already presented in section 7 and 8 and explain how the Linbland-Kossakowski form of the relaxation superoperator for the model free approach (14) can be conveniently calculated. The Linbland-Kossakowski form of the relaxation superoperator is given by (14):

$$
\begin{aligned}
-\hat{\Gamma}_{0} \sigma & =\sum_{s \neq s^{\prime}} \Gamma_{s s^{\prime}}\left[O_{s s^{\prime}} \sigma O_{s^{\prime} s}-\frac{1}{2}\left(\sigma O_{s^{\prime} s^{\prime}}+O_{s^{\prime} s^{\prime}} \sigma\right)\right]+ \\
& +\sum_{q} \Gamma_{q}\left[O_{q} \sigma O_{q}-\frac{1}{2}\left(\sigma O_{q} O_{q}+O_{q} O_{q} \sigma\right)\right] .
\end{aligned}
$$

Where $\hat{\Gamma}_{0}$ indicates a matrix of the relaxation superoperator for the model-free approach, $O_{s s^{\prime}}=v_{s} v_{s}^{*}$ are the eigenoperators constructed from the eigenvectors of the stationary Hamiltonian in the Hilbert space. The requirement that the operators $O_{s s}$ are traceless can be fulfilled by setting up a linear combination of the former: $O_{q}=\sum_{s=1}^{N} c_{q s} O_{s s}$. The coefficient $c_{q s}$ are found by solving a set of equations given in (15). To avoid these cumbersome calculations we can further simplify (14), the second term can be rewritten in the non-diagonal form:

$$
\begin{gathered}
\sum_{q^{\prime}} \Gamma_{q}\left[O_{q} \sigma O_{q}-\frac{1}{2}\left(\sigma O_{q}+O_{q} \sigma\right)\right]= \\
\sum_{s s^{\prime}} \bar{\Gamma}_{s s^{\prime}}\left[O_{s s} \sigma O_{s^{\prime} s^{\prime}}-\frac{1}{2}\left(\sigma O_{s^{\prime} s^{\prime}} O_{s s}+O_{s^{\prime} s^{\prime}} O_{s s} \sigma\right)\right]= \\
\sum_{s \neq s^{\prime}} \bar{\Gamma}_{s s^{\prime}} O_{s s} \sigma O_{s^{\prime} s^{\prime}}+ \\
\sum_{s} \bar{\Gamma}_{s s}\left[O_{s s} \sigma O_{s s}-\frac{1}{2}\left(\sigma O_{s s}+O_{s s} \sigma\right)\right]
\end{gathered}
$$

where the rates $\bar{\Gamma}_{s s^{\prime}}$ are expressed via $\Gamma_{q}$ and $c_{q s}$. It follows from the form of (14) that:

$$
\hat{\Gamma}_{0} O_{k k}=\sum_{s \neq k} R_{1, s k}\left(O_{k k}-O_{s s}\right), \quad \hat{\Gamma}_{0} O_{k j}=R_{2, k j} O_{k j}, \quad k \neq j
$$

which in terms of the rates $\bar{\Gamma}_{s s^{\prime}}$, and gives us:

$$
R_{2, k j}=\frac{1}{2}\left(\bar{\Gamma}_{k k}+\bar{\Gamma}_{j j}-2 \bar{\Gamma}_{k j}+\sum_{s \neq k} \Gamma_{s k}+\sum_{s \neq j} \Gamma_{s j}\right), \quad R_{1, s k}=\Gamma_{s k}
$$

If the rates $R_{1, s k}, R_{2, k j}$ are known one can invert the relation in (30) to find $\bar{\Gamma}_{s s^{\prime}}$ and $\Gamma_{s s^{\prime}}$, letting further $\bar{\Gamma}_{s s^{\prime}}=0$ one gets:

$$
\Gamma_{s k}=R_{1, s k}, \quad \bar{\Gamma}_{k j}=\frac{1}{2}\left(\sum_{s \neq k} R_{1, s k}+\sum_{s \neq j} R_{1, s j}\right)-R_{2, k j}
$$


The rates $R_{1, s k}, R_{2, k j}$ can be found from the projection of the eigenoperators $O_{k j}$ :

$$
R_{2, k j}=\operatorname{Tr}\left[\left(\hat{\Gamma} O_{k j}\right) O_{j k}\right], \quad R_{1, s j}=-\operatorname{Tr}\left[\left(\hat{\Gamma} O_{k k}\right) O_{s s}\right],
$$

where $\hat{\Gamma}$ is the relaxation superoperator matrix for the uncorrelated random field model given by (10). To calculate the projection, operators $O_{k j}$ have be represented column wise as vectors. The trace is taken if the product of $\hat{\Gamma} O_{k j}$ is transform back the operator representation or the scalar product if $O_{j k}$ is in the vector form. Finally the following expression for the relaxation superoperator matrix of the model-free approach is obtained:

$$
\begin{aligned}
-\hat{\Gamma}_{0} \sigma=\sum_{s \neq s^{\prime}} \Gamma_{s s^{\prime}} & {\left[O_{s s^{\prime}} \sigma O_{s^{\prime} s}-\frac{1}{2}\left(\sigma O_{s^{\prime} s^{\prime}}+O_{s^{\prime} s^{\prime}} \sigma\right)\right]+} \\
& +\sum_{s \neq s^{\prime}} \bar{\Gamma}_{s s^{\prime}} O_{s s} \sigma O_{s^{\prime} s^{\prime}}
\end{aligned}
$$

The advantage of the form (33) is that it contains only the operators $O_{s s^{\prime}}$ and rates $\Gamma_{s s^{\prime}}, \bar{\Gamma}_{s s^{\prime}}$ easily calculated from (31) and (32).This form is especially convenient if operators $O_{s s^{\prime}}$ are expressed as the left/right superoperators (operators in Liouville space) i.e. $\hat{O}_{s s^{\prime}}^{L}=O_{s s^{\prime}} \otimes 1, \hat{O}_{s s^{\prime}}^{R}=1 \otimes O_{s s^{\prime}}^{*}$ It is worth to notice that (33) gives the thermalize form of the relaxation superoperator thus the homogenous form of the master equations is preserved and exponential solution can be used. Above-mentioned treatment can be adopted in one of the available spin dynamics simulation software package .e.g Spinach. 